\title{
Procedimiento para la gestión del turismo académico (grupos) en la oficina coordinadora de servicios académicos (OCSA) de la Universidad Central "Marta Abreu" de las Villas.
}

Procedure for the management of academic tourism (groups) at the coordinating office for academic services (OCSA) in Universidad Central "Marta Abreu" de las Villas.

Lic. Isis Laura Corcho Reyes. ${ }^{1} \&$ MsC Luis Miguel Campos Cardoso. ${ }^{2}$

\section{Resumen.}

Como parte del turismo académico se consideran las estancias temporales de estudiantes, profesores, eventistas, cursantes, aspirantes y turistas en instituciones de educación superior, fuera de sus lugares de residencia habitual. Impulsado por diferentes programas de intercambio esta nueva tipología de turismo ha experimentado un crecimiento muy importante en los últimos años, reportando ingresos considerables a las instituciones de educación superior. El turismo académico es una tipología turística contemporánea, que ha emergido debido al aumento de los desplazamientos, efecto de la internacionalización de la educación superior. Esta tipología es compleja y no hay que entenderla sólo desde la perspectiva estudiantil sino también desde una multiforme gama de aristas y multimotivaciones que puedan tener las personas que se desplazan a instituciones de Educación Superior (pasantías, cursos, eventos, doctorados, maestrías, visitas guiadas, etc.). De esta forma se demandan procedimientos y metodologías que permitan gestionar esta modalidad de forma asertiva y coherente, como alternativa sostenible para instituciones de Educación Superior. El procedimiento para la gestión del Turismo Académico en la UCLV es una herramienta que contribuye resolver los problemas que emergen en el proceso de gestión. De su aplicación se deriva el diagnóstico de la gestión de la modalidad, a partir de lo cual se plantea la estrategia de gestión en la universidad y

\footnotetext{
${ }^{1}$ Universidad Central "Marta Abreu" de Las Villas, Departamento de Turismo, Cuba, icorcho@uclv.cu

${ }^{2}$ Universidad Central "Marta Abreu" de Las Villas; Oficina Coordinadora de Servicios Académicos (OCSA); Dirección de Relaciones Internacionales, Cuba, lmcampos@uclv.edu.cu
} 
objetivos estratégicos a fin de disponer de una guía planificada, coordinada, flexible y con fácil aplicación para instituciones universitarias, sedes de eventos, académicos y otros entes interesados, asociadas a la gestión del turismo académico.

Palabras claves: turismo, turismo académico, internacionalización, educación superior.

\section{Abstract.}

As part of the academic tourism, the temporary stays of students, professors, event participants, students, aspirants and tourists in institutions of higher education are considered, outside of their places of habitual residence. Driven by different exchange programs this new type of tourism has experienced a very important growth in recent years, reporting considerable income to higher education institutions. Academic tourism is a contemporary tourism typology, which has emerged due to the increase in displacements, the effect of the internationalization of higher education. This typology is complex and should not be understood only from the student perspective but also from a multiform range of edges and multimotivations that may have people who move to higher education institutions (internships, courses, events, doctorates, masters, guided tours , etc.). In this way, procedures and methodologies are required to manage this modality in an assertive and coherent manner, as a sustainable alternative for Higher Education institutions. The procedure for the management of Academic Tourism in the UCLV is a tool that helps to solve the problems that emerge in the management process. The diagnosis of management of the modality derives from its application, from which the management strategy in the university and strategic objectives is proposed in order to have a planned, coordinated, flexible and easy-to-apply guide for university institutions. , venues of events, academics and other interested entities, associated with the management of academic tourism.

Keywords: tourism, academic tourism, internationalization, higher education.

\section{Introducción.}

El fenómeno turístico se ha perfilado, a partir de la segunda mitad del siglo XX, con énfasis particular en la denominada Nueva Era del Turismo, como la más dinámica actividad económica a nivel mundial e importante impulsor del desarrollo, en continua expansión y diversificación. El turismo internacional representa en la actualidad el $9 \%$ del Producto Interno Bruto (por efecto indirecto e inducido); el 6\% de las exportaciones mundiales con 1.3 billones de dólares; genera 1 de cada 11 empleos y es fuente imprescindible de desarrollo local y regional.

El año 2017 se caracterizó por un crecimiento sostenido en muchos destinos y la firme recuperación de los destinos que sufrieron declives en años previos. Los resultados se debieron en parte a la recuperación económica y a la fuerte demanda de numerosos mercados emisores tradicionales y emergentes, así como, en particular, al repunte del gasto turístico en Brasil y la Federación de Rusia después de varios años de descensos. Los grandes y sucesivos avances científico-tecnológicos, en la educación, la cultura, el deporte y todas las ramas del saber conllevan a la necesidad constante del intercambio y ello al crecimiento de estos eventos a escala mundial en sus diferentes tipologías. Según 
Pawloska (2012) la movilidad universitaria internacional ha experimentado un fuerte crecimiento en los últimos años, contando en la actualidad los centros de educación superior con un porcentaje creciente y cada vez más importante de alumnos procedentes de otros países. A nivel mundial la movilidad entre las universidades se ha visto favorecida por una serie de factores entre los que se encuentran los numerosos convenios firmados entre las mismas, la inclusión dentro de la oferta de estas de cursos destinados exclusivamente a estudiantes extranjeros o la potenciación de distintos programas de intercambio.

En este trabajo se utiliza el término turismo académico para hacer referencia a todas las estancias de duración inferior a un año, realizadas en centros de educación superior situados fuera del entorno habitual de residencia del estudiante. Al igual que se distingue entre turismo internacional e interno, se podría hablar de turismo académico internacional para hacer referencia a los movimientos de universitarios entre distintos países y de turismo académico doméstico, que incluiría los movimientos de universitarios nacionales dentro del propio país. El turismo académico internacional receptor, siguiendo la definición apuntada, incluiría las actividades realizadas por los estudiantes universitarios procedentes de otros países en sus estancias de duración inferior a un año. Esta movilidad incluye básicamente los desplazamientos que se realizan en el marco de los diferentes programas de intercambio y aquellos que tienen lugar con el objetivo de aprender o perfeccionar el idioma, entre otros (Tomado a partir del Martínez, 2012). En Cuba, estos flujos o movimientos turísticos cuyo motivo principal de viaje está relacionado con lo académico ha experimento un crecimiento muy importante en los últimos años en la medida que las universidades han diversificado sus estrategias de internacionalización adaptándolas a las actuales condiciones mundiales, así como el aumento de los flujos de profesionales y estudiantes son cada vez mayores.

A pesar de todos los esfuerzos que las instituciones universitarias del país realizan en ampliar su cartera de productos académicos así como de fomentar los servicios académicos como una vía sostenible de aumentar los ingresos netos a la universidad, se perciben algunas descoordinaciones por parte de los gestores de esta modalidad turística, no se realizan acciones coordinadas con los turoperadores especializados de esta modalidad y en muchas ocasiones no se comprende la necesidad de aplicar herramientas científicamente fundamentadas para gestionar la modalidad de turismo académico de una forma planificada, organizada y coherente. El objetivo general de esta investigación está orientado a compartir la propuesta de un diseño de un procedimiento que permita la gestión del Turismo Académico en la Universidad Central "Marta Abreu" de Las Villas.

\section{Métodos.}

La revisión bibliográfica permitió identificar una serie de procedimiento acerca del turismo de Eventos e incentivos a partir de los cuales se establece la nueva propuesta para la gestión del turismo académico.

Para el diseño del procedimiento, se parte de la comparación realizada de las diversas herramientas estudiadas que, aunque no son abordadas explícitamente en esta memoria, es el resultado de la investigación que han realizado los investigadores conjuntamente con Delgado (2015), Peñalver (2015) y Bermudez (2013), aspecto que sienta las bases para formular las etapas, fases y pasos que componen la propuesta. Además, se considera 
la experiencia que existe hasta el momento en el desarrollo de la actividad. El procedimiento propuesto se retoma a partir del procedimiento de Peñalver (2015) y el de Bermúdez (2013) y se adecúa para la modalidad de Turismo Académico.

Figura 1. Diagrama de fases.

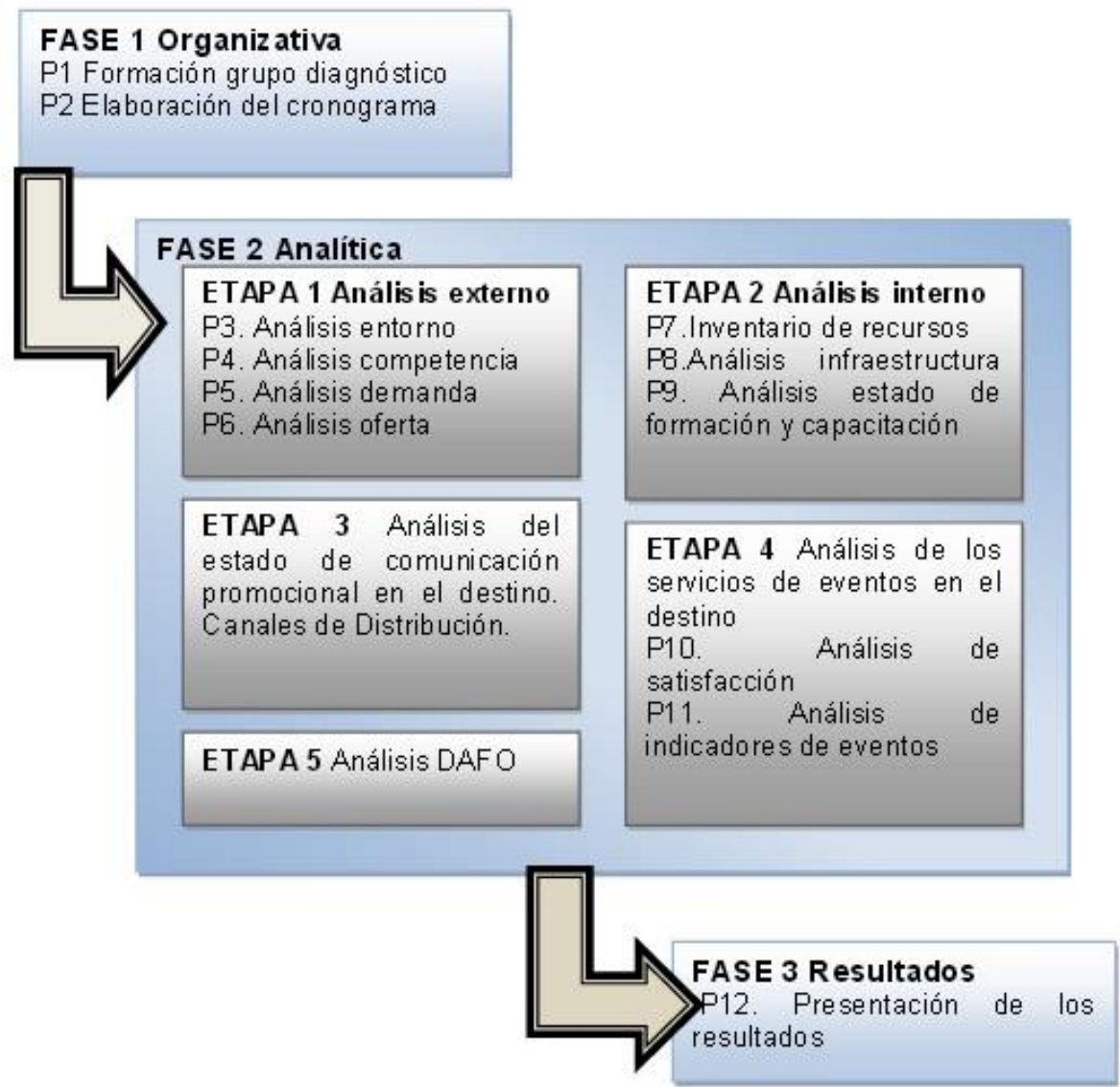

\section{Resultados.}

Martínez (2012) considera la gestión como un sistema que comprende desde la previsión, planeación y organización hasta la integración, mando o dirección y control. Justamente estas actividades gerenciales constituyen la base de la gestión de eventos e incentivos y también del Turismo Académico, teniendo en cuenta, además, las aéreas de resultado claves (Cruz y Rodríguez, 2005) para la modalidad ajustadas al destino/entidad en que se aplique. La eficiencia de la gestión, condicionara infaliblemente el desarrollo de los eventos en un destino turístico.

Basado en esto, y una vez detectadas las necesidades del destino, se considera que la elaboración de un procedimiento para la gestión del Turismo Académico da la posibilidad de, en primer lugar, detectar las problemáticas actuales de la gestión en el destino o en el centro de educación superior que se pretenda aplicar y luego plantear las estrategias de gestión y objetivos estratégicos respondiendo a las áreas de resultados claves para la modalidad, que sirvan de referencia a su proyección y desarrollo futuro. 
I Fase Organizativa: De ella depende en gran medida el avance del proceso de diagnóstico. Es la fase en la que se definen los miembros del equipo de análisis, los cuales tendrán como base la participación, proceso activo encaminado a transformar las relaciones de poder, alcanzando distintos niveles y diferentes formas de expresión (Gianoten y Wit citado en Fals et al., 2011).

\section{Paso 1. Formación del grupo de trabajo}

\section{Paso 2. Elaboración del cronograma de trabajo}

\section{Fase Analítica:}

Diagnóstico de la Gestión de Turismo Académico: Aquí se retoma, adecuado a las características de la presente propuesta, el procedimiento propuesto por Bermúdez (2013) a partir de la Fase 2, pues luego de su análisis, se considera que es el más completo concebido hasta el momento para el diagnóstico de esta modalidad turística.

Etapa 1. Análisis externo Comprende el estudio del mercado turístico, la competencia, el entorno y sus implicaciones para desarrollar la modalidad en un destino/institución de educación superior.

Paso 3. Análisis del entorno El análisis del entorno parte del estudio de los factores no controlables a nivel mundial, de país y de destino que limitan o impulsan su desarrollo:

Paso 4. Análisis de la competencia: Asumiendo como competidores aquellos que venden productos y servicios similares al destino en cuestión (ESEM, 2010), se recomienda realizar un análisis de los posibles destinos competidores relacionados con el Turismo Académico a nivel internacional y a nivel interno. Identificar países y ciudades con crecimientos acelerados. Para ello se propone aplicar la propuesta de análisis de competencia que realiza Acerenza (2004) con algunas adecuaciones que favorezcan su adaptación al objeto de estudio, considerando los criterios: cantidad de eventos académicos realizados, naturaleza del producto, precios públicos, ingresos turísticos y cantidad de participantes.

Paso 5. Análisis de la demanda: Se considera el análisis de la demanda actual y potencial. Se analizan principales mercados emisores turísticos a nivel de país y de destino. Se caracteriza el mercado de la modalidad a partir del procedimiento de segmentación de Cobra (2000) que parte de la definición de variables, desarrollar un modelo de agrupación, llevar a cabo el montaje de los segmentos, para declarar estrategias.

Paso 6. Análisis de la oferta: Una vez que se conoce que la oferta es la combinación del producto con el precio, se deben detectar durante este paso las principales tendencias, y factores claves de éxito que se puedan convertir en oportunidades y amenazas para el destino/institución de educación superior. Se debe valorar si el producto turístico académico funciona de manera integrada a partir de la sinergia que provoca entre la sede generadora (universidad, ONG, institución, etc.), sede que lo organiza, el comité 
organizador, hoteleros, los restaurantes, transportistas y otros entes de la actividad turística.

Etapa 2. Análisis interno Los destinos turísticos o instituciones de educación superior deben realizar un análisis interno para identificar y evaluar sus recursos turísticos, a fin de asegurar que el destino/institución objeto de investigación aproveche y ofrezca una combinación atractiva de productos a su mercado objetivo.

Paso 7. Identificación y análisis de los recursos turísticos En la presente investigación se asume por recurso turístico: los elementos disponibles en un espacio dado, ya sean, de origen natural o creados por el hombre, susceptibles de su uso y aprovechamiento con fines turísticos (Colectivo de autores, 2012).

Paso 8. Análisis de la infraestructura: La infraestructura para el desarrollo de la modalidad de turismo académicos resulta decisiva en el desarrollo de la misma, por lo que es necesario determinar sus condiciones a través del inventario de infraestructuras propias para la modalidad, dígase: universidades con infraestructura para eventos académicos (aulas, salones, teatros, laboratorios, aulas especializadas, etc.) salones de reuniones, centros de ferias y exposiciones, hoteles con infraestructura para eventos académicos, sedes de eventos. En el desarrollo del análisis de la infraestructura se comprende: el estado técnico (localización de la sede, organismo a que pertenece, cumplimiento de las normas para sedes de eventos académicos); el equipamiento (mobiliario, computadoras, cabinas, tecnología específica para eventos académicos) etc.

Paso 9. Análisis del estado de la formación y la capacitación: Analizar la disponibilidad de entrenamientos u otras acciones de capacitación a través de cursos, postgrados, diplomados y especialidades. Analizar la formación de personal relacionado con la actividad comprendida dentro de la modalidad de turismo académico (gestores, OPC, intérpretes etc.)

Paso 10. Análisis de la variable comunicación promocional para la modalidad de turismo académico en el destino.

Valorar el estado actual de acciones de promoción, publicidad, promoción de ventas, relaciones públicas, y el marketing directo, al conocer que todas las acciones relacionadas con el Turismo de Eventos e Incentivos (como marca paraguas que agrupa al turismo académico), en su sentido más amplio, constituyen acciones de comunicación social. Para ello se especifica que se entiende por cada acción que integra la mezcla promocional según Benavides (2000):

La publicidad: Es impersonal porque utiliza los medios de comunicación masiva en la transmisión de mensajes que estimulan el deseo por el producto. En los eventos académicos las formas más utilizadas son la preparación de carteles o afiches, anuncios en publicaciones, la prensa, radio, televisión, Internet, displays, vallas. 
Paso 11. Análisis de los servicios académicos: Se concreta a través del Análisis de Satisfacción: Resulta importante conocer criterios de guías, participantes y líderes de opinión de los eventos más representativos del destino turístico, así como de las visitas guiadas, conferencias especializadas, criterios de participantes a eventos académicos según experiencia demostrada, para poder resumir en breve valoración la calidad de los servicios académicos. En base a ello se conciben la encuesta como herramienta para conocer el nivel de satisfacción de los involucrados.

Paso 12. Análisis de los indicadores del Turismo Académico: Los indicadores son instrumentos que permiten recoger de manera adecuada y representativa, información habitualmente numérica de la actividad respecto a la ejecución de uno o varios procesos. Los indicadores propuestos incluyen tanto eventos como servicios académicos que enmarcan la modalidad analizada:

- Número de eventos en el destino,

- Total, de participantes (nacionales y extranjeros),

- Mercados emisores,

- Sedes de eventos,

- Estacionalidad de los eventos,

- Número de eventos según temáticas,

- Número de eventos según su carácter (diseño propio o sede captada),

Número de eventos cuya tipología no son comprendidos por el BCC

- Número de cursos cortos

- Cantidad de pasantías

- Cantidad de visitas guiadas

- Cantidad de carreras completas

- Cantidad de maestrías (contra edición en ejecución)

- Doctorados curriculares o tutoriales (cursando)

- Cantidad de conferencias especializadas

Principales mercados que solicitan estos servicios

Principales mediadores (AA. VV)

\section{Etapa 3. Análisis DAFO}

\section{Fase. Estratégica}

Gestión del Turismo Académico: Los resultados del diagnóstico de la gestión de eventos e incentivos, son vitales para la fase estratégica, como función fundamental en la gestión. 
Etapa 4. Definición del problema estratégico: El problema estratégico se determina a partir de los elementos que resultan más reiterativos en el análisis de la matriz DAFO. Es la definición del problema o brecha entre el estado real del destino y el estado deseado, así como su gran solución (Rodríguez, 2013).

Etapa 5. Definición del propósito estratégico: Aquí se considera de especial importancia la definición del propósito de la gestión de turismo académico en el mediano y largo plazo; se determinan la estrategia maestra principal y de ahí estrategias específicas para el cumplimiento de los objetivos estratégicos de forma que el destino alcance su misión y su visión (Herrera, 2000).

Paso 13. Definición de la Misión: La Misión, propósito o razón de ser, pueden admitirse como similares siempre que quede claro que se está refiriendo a la finalidad global del destino, a aquello que no puede dejar de hacer porque de lo contrario sería otra cosa (Jarrillo, 1996; Díaz, C.; Koontz y Weihrich, 2011; Martínez, 2012; Carballo, E. y J. Rodríguez, 2013).

Paso 14. Definición de la Visión. "La visión no es más que una imagen razonablemente clara de un futuro deseable, es la capacidad para imaginar futuros alternativos... es tanto una actitud como un estilo de pensamiento, una disposición a considerar más posibilidades de cambio.” (Rodríguez, 2013).

Paso 15. Valores compartidos: Los Valores Compartidos representan las conductas que los trabajadores, especialistas y dirigentes del destino relacionados con la modalidad deben seguir para el logro de los objetivos, es decir, determinan en el orden táctico, el comportamiento para llegar a la Visión (Herrero, 2010; Portuondo, 2011).

Paso 16. Previsión de escenarios (Análisis prospectivo) Por escenarios se entiende la descripción de los posibles marcos en los que debe funcionar el turismo de en el destino y que ejercen influencia en su comportamiento (Wildblood, 2001; Tena, 2000).

Paso 17. Formulación estratégica del Turismo Académico: En este paso se propone el Planteamiento de la Estrategia General de Gestión del turismo Académico. La estrategia es un medio de establecer los propósitos organizativos en términos de objetivos a largo plazo y programas de adopción de cursos de acción (Matos, 2005).

Etapa 6. Proyección estratégica: Esta etapa se encarga de traducir a acciones concretas, la visión estratégica de la entidad, es decir, convertir la visión de la institución en resultados claros y medibles de desempeño de la actividad de eventos académicos, que se deberá lograr en el largo plazo, con metas intermedias a mediano plazo (Menguzzato y Renau, 1995; Portuondo, 1998; Martínez, 2012; Molina, 2013).

Paso 18. Determinación de las áreas de resultados clave (ARC): Las ARC son aquellas donde el desempeño es vital para el desarrollo de la institución a más largo plazo, por lo que tienen que determinarse y a partir de ellas fijar objetivos verificables (Herrera, 2000). 
Se retoman las aéreas de gestión claves para la modalidad de turismo de eventos e incentivos, definidas por Cruz y Rodríguez (2005), Rodríguez (2013) y propuestas por Peñalver (2015), ajustadas a los turismos académicos y aplicables a instituciones donde se desarrolla el Turismo Académico, ente las que se encuentran:

- Gestión de servicios académicos: enfocada en la estructura legal, administración, operaciones y políticas a las cuales debe regirse la actividad de eventos académicos. Incluye además la implementación de procedimientos, metodologías y normas para la organización de eventos académicos, la elaboración del calendario de eventos, selección a temáticas, tecnologías y servicios especializados, lo que permite ampliar el mercado generador y consumidor de eventos en beneficio del destino.

- Gestión de sedes académicas: encargada de la gestión de infraestructura, facilidades, equipamiento y tecnología. Es el área que garantiza la tecnología y el correcto funcionamiento del sistema informativo en instalaciones y sedes. Permite a su vez la elaboración de estadísticas y el procesamiento y análisis de la información de los indicadores que miden el comportamiento de la actividad de eventos académicos.

- Gestión de los recursos humanos: actúa sobre la disponibilidad de formación de OPC y personal especializado en atender esta modalidad. La gestión de cursos de capacitación e inversiones en personal especializado en servicios a eventos académicos; política y cultura organizacional.

- Gestión económico-financiera: enfocada a las políticas financieras y procedimientos, contratación, presupuesto de operación, fuentes de financiamiento, administración de los ingresos; proceso de planificación y control de todas las actividades contables y financieras, así como los resultados económicos, la rentabilidad y las proyecciones futuras del turismo académico en instituciones de educación superior, que permita a gestores de la actividad, tomar decisiones.

- Gestión de sedes generadoras de turismo académico: Las sedes generadoras son las que originan, crean, generan los eventos académicos.

- Gestión de la comercialización: Enfocado a variables Producto, Precio y Distribución; encaminada a establecer acciones concretas para la comercialización efectiva de la oferta exportable de servicios académicos, así como eventos del destino en el mercado del turismo académico. Contratación, grupos FAM.

- Gestión de la comunicación promocional para eventos académicos: tener en cuenta los aspectos relacionados con el mix de comunicación de marketing: Relaciones Públicas, Publicidad, Merchandising, marketing directo, promoción de ventas, ventas directas.

- Gestión de la Calidad de los servicios a eventos académicos 
- Gestión de actividades complementarias

Paso 19. Determinación de Objetivos Estratégicos por ARC y Estrategias por Objetivos: Los Objetivos Estratégicos por ARC generalmente abarcan un ARC y se formulan a partir de los resultados del diagnóstico estratégico, de la misión, la visión y los valores de la empresa (Herrera et al., 2001).

\section{Fase operativa. Presentación de los resultados}

Paso 20. Confección del informe final: Confección de un informe detallado de los resultados del estudio: la aplicación del procedimiento, con sus fases, etapas y desarrollo, con especial énfasis en: el diagnóstico de la gestión del turismo académico, de cuyos resultados se nutrirá la fase estratégica, que debe registrarse en detalle, en especial la estrategia de gestión académica definida por la institución y su proyección estratégica a modo de objetivos por ARC y estrategias por objetivos.

\section{Fase de control.}

\section{Paso 21. Confección del Informe Final e Implementación de la estrategia}

Paso22. Control, retroalimentación y seguimiento: El control del diagnóstico permite que se puedan realizar los ajustes necesarios a la estrategia general de gestión o a los objetivos estratégicos y estrategias, según la experiencia práctica, en las condiciones y el momento que el destino demande (Mintzberg, 2010; Martínez, 2012). Para la retroalimentación y el seguimiento de la aplicación del procedimiento en el destino, los expertos recomiendan al equipo de trabajo diseñar un Plan de acción.

\section{Conclusiones.}

$>$ El procedimiento de Gestión del Turismo Académico constituye en la actualidad una necesidad para contribuir a una mejor gestión, que se mide en altos ingresos y dinámicos flujos turísticos, en medio de un entorno altamente competitivo, lo cual ha elevado el interés de académicos y otros organismos implicados en el desarrollo de eventos académicos.

$>$ Se realiza sobre la base de los aspectos positivos sobresalientes en el análisis comparativo de las metodologías propuestas por los autores estudiados, considerando, a su vez, el carácter sistémico del desarrollo turístico que genera relaciones entre el producto y los elementos del marco en el que se desarrolla la modalidad.

$>$ Se destaca por su estructura detallada y comprensible para los usuarios, lo cual permite su inserción en el contexto turístico, lo que resalta el carácter práctico del procedimiento como instrumento de análisis estratégico de un negocio tan rentable como lo es el turismo académico y su aplicación integral, constituye una herramienta estratégica de alto valor para la gestión comercial del turismo especializado en instituciones de educación, aplicable a cualquier tipo de institución o entidad que desarrolle esta modalidad turística. 


\section{Referencias Bibliográficas.}

OMT enero 2018 Resultados del turismo internacional en 2017: los más altos en siete años http://media.unwto.org/es/press-release/2018-01-15/resultados-del-turismointernacional-en-2017-los-mas-altos-en-siete-anos

Bermúdez Zamora, A. (2013). Procedimiento para el diagnóstico estratégico del Turismo de Eventos e Incentivos en el destino turístico Villa Clara. Tesis en opción al grado científico de Máster en Gestión Turística. Universidad Central Marta Abreu de Las Villas. Santa Clara, Villa Clara, Cuba.

Campos, L. M., (2010). Dossier de Organización Profesional de Eventos. Cuba, Centro de Estudios Turísticos, UCLV. Santa Clara, Cuba.

Martínez, F., (2012) Planeación Estratégica Creativa. Editorial PAC. México.

Pawloska, E. (2012). El turismo académico en Galicia: otra forma de contribución de las universidades a las economías locales. Revista Cuaderno de Turismo no 32. Universidad de Murcia. España.

Peñalver Medina, Y. (2015). Procedimiento para la gestión del Turismo de Eventos e Incentivos. Aplicación al destino turístico Villa Clara. Tesis en opción al grado científico de Máster en Gestión Turística. Universidad Central Marta Abreu de Las Villas. Santa Clara, Villa Clara, Cuba.

Organización Mundial de Turismo, (2013) Panorama de turismo internacional, Departamento de Publicaciones. España.

Soto Leiva, F. (2012). La movilidad estudiantil internacional como turismo académico. Revista Geografía Valparaíso. No 42 Año 2012. ISSN 0718-9877. Valparaíso, Chile.

\section{LCiencia}




\section{Para citar el artículo indexado.}

Corcho I. \& Campos L. (2019) Procedimiento para la gestión del turismo académico (grupos) en la oficina coordinadora de servicios académicos (OCSA) de la Universidad Central "Marta Abreu” de las Villas. Revista electrónica Explorador Digital 3(2), 48-59. Recuperado desde:

http://cienciadigital.org/revistacienciadigital2/index.php/exploradordigital/article/view/419/9 $\underline{56}$

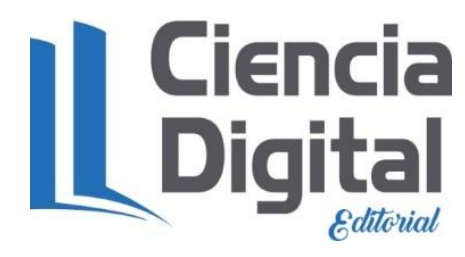

El artículo que se publica es de exclusiva responsabilidad de los autores y no necesariamente reflejan el pensamiento de la Revista Explorador Digital.

El articulo queda en propiedad de la revista y, por tanto, su publicación parcial y/o total en otro medio tiene que ser autorizado por el director o editor de la Revista Explorador Digital.
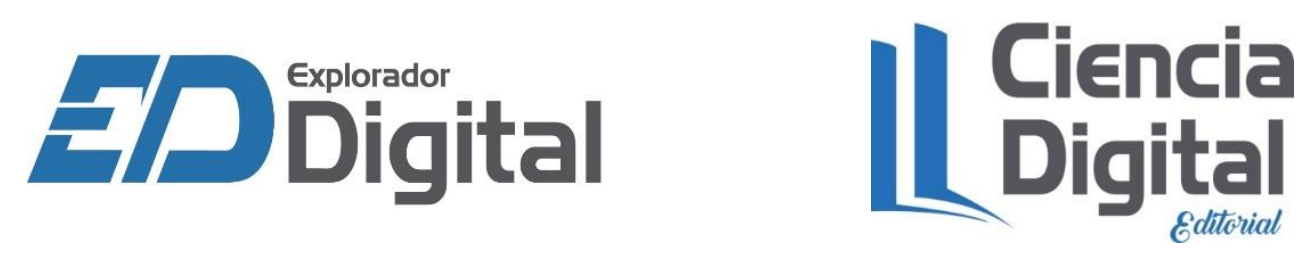